\title{
Useful Identities for Half-Space Problems in Linear Transport Theory*
}

\author{
R. E. Aamodt $\dagger$ and K. M. Case \\ Department of Physics, The University of Michigan, Ann Arbor, Michigan
}

A typical translationally invariant linear transport problem for a halfspace is solved by the eigenfunction method. Using some derived identities for the solution of the associated homogeneous Hilbert problem it is shown that the usual cumbersome integrals occurring in half-space problems can be reduced to a simple form. The usefulness of the identities is illustrated by solving the problem of a plasma confined to a half-space by a diffuse reflecting wall.

\section{INTRODUCTION}

A large class of problems in linear transport phenomena can be formulated as boundary value problems and solved by the eigenfunction technique $(1-6)$. Typically, the governing equation possesses translational invariance and can be reduced to the form:

$$
\left(\mu \frac{\partial}{\partial x}+1\right) \psi(x, \mu)=f_{1}(\mu) \int_{-\beta}^{\beta} f_{2}\left(\mu^{\prime}\right) \psi\left(x, \mu^{\prime}\right) d \mu^{\prime},
$$

where $-\beta \leqq \mu \leqq \beta$, and $f_{1}(\mu) \cdot f_{2}(\mu)$ is a real even function of $\mu$. We first show in this paper that with suitable restrictions on $f_{1}(\mu) \cdot f_{2}(\mu)$, Eq. (1.1) always generates a complete set of solutions. Using these solutions, some typical halfspace problems are considered and it is shown that some identities established for the solution of the associated homogeneous Hilbert problem allow the quantities of physical interest to be expressed in a simple form. To illustrate the usefulness of these identities the problem of a plasma confined to a half-space by a diffuse reflecting wall is then solved.

II. SOLUTIONS OF EQ. (1.1)

The translational symmetry of Eq. (1.1) suggests looking for solutions of the form:

$$
\psi_{\nu}(x, \mu)=e^{-x i \nu} \phi_{\nu}(\mu) .
$$

* Supported in part by the Office of Naval Research, Department of the Navy.

$\dagger$ Present address: John Jay Hopkins Laboratory for Pure and Applied Science, Generul Atomic Division of General Dynamies Corporation, San Diego, California. 
For $\nu$ finite (1.1) becomes:

$$
(\nu-\mu) \phi_{y}(\mu)=\nu f_{1}(\mu) \int_{-\beta}^{\beta} f_{2}\left(\mu^{\prime}\right) \phi_{\nu}\left(\mu^{\prime}\right) d \mu^{\prime} .
$$

Choosing the convenient normalization of $\phi_{p}(\mu)$;

$$
\int_{-\beta}^{\beta} f_{2}(\mu) \phi_{\nu}(\mu) d \mu=1,
$$

Eq. (2.2) can be solved by the standard procedure (2). The usual discrete eigenvalues are then given by the zeros of $\Lambda(\nu)$, where

$$
\Lambda(\nu) \equiv 1-\int_{-\beta}^{\beta} f_{2}(\mu) \phi_{\nu}(\mu) d \mu=1+\nu \int_{-\beta}^{\beta} \frac{f_{1}(\mu) f_{2}(\mu) d \mu}{\mu-\nu} .
$$

We will always assume that $f_{1}(\mu) \cdot f_{2}(\mu)$ belongs to the class $H^{*}(\gamma)$ on $(-\beta, \beta)$, for $\beta$ finite; and for $\beta$ infinite that $f_{1}(\mu) \cdot f_{2}(\mu)$ belongs to the class $H$ and satisfies:

$$
\operatorname{Lim}_{|\mu| \rightarrow \infty} f_{1}(\mu) \cdot f_{2}(\mu) \leqq C|\mu|^{-(2+\alpha)}, \quad \alpha>0 .
$$

Then, $\Lambda(\nu)$ is a real even function of $\nu$, sectionally holomorphic with boundary $(-\beta, \beta)$, and asymptotically,

$$
\Lambda(\nu) \underset{\nu \rightarrow \infty}{\simeq} 1-\int_{-\beta}^{\beta} f_{1}(\mu) f_{2}(\mu) d \mu+0\left(\frac{1}{\nu^{2}}\right) .
$$

Clearly if $\nu_{i}$ is a root of $\Lambda(\nu)=0$, so is $-\nu_{i}$ and $\pm \nu_{i}^{*}$. Defining $\nu_{-i}=-\nu_{i}$, it will be assumed (only for convenience) that the $\nu_{i}$ can be labeled so that $\operatorname{Re} \nu_{i}>0, i>0$, and if $\nu_{i+1}^{*} \neq \nu_{i}$, then $\left|\operatorname{Re} \nu_{i}\right|<\left|\operatorname{Re} \nu_{i+1}\right|$.

For $\nu$ not on the interval $(-\beta, \beta) \Lambda(\nu)$ will in general have $2 N^{\prime}$ zeros, where $N^{\prime}$ includes the order of the zero. That is:

$\Lambda^{(j-1)}\left(\nu_{i}\right)=\left.\frac{d^{(j-1)}}{d \nu^{(j-1)}} \Lambda(\nu)\right|_{\nu=\nu_{i}}=0 \quad j=1,2, \cdots m_{i}, \quad \pm i=1,2, \cdots n^{\prime}$

and

$$
N^{\prime}=\sum_{i=1}^{n^{\prime}} m_{i}
$$

In tins paper it is required that $\Lambda_{ \pm}(\nu) \neq 0$ for $\nu$ on the interval $[-\beta, \beta]$. (As usual, $\Lambda_{+}(\nu)$ and $\Lambda_{-}(\nu)$ denote the boundary values of $\Lambda(\nu)$ from above and below the cut respectively.) This condition can be relaxed but it considerably complicates the results of this paper without furthering its content. With this requirement and choosing the $\arg \Lambda_{+}(0)=0$, the usual change of argument theorem gives us:

$$
N^{\prime}=\frac{1}{\pi} \Delta_{(0, \beta)} \arg \Lambda_{+}(\nu)=\frac{1}{\pi} \arg \Lambda_{+}(\beta) .
$$


$\left(\Delta_{(L)} \equiv\right.$ change along $\left.L\right)$. As $\operatorname{Im} \Lambda_{+}(\nu)=\nu \pi f_{1}(\nu) f_{2}(\nu)$, if $f_{1}(\nu) \cdot f_{2}(\nu)$ has $M$ zeros for $0<\nu \leqq \beta$, clearly $N^{\prime} \leqq M+1$. We will always assume that $N^{\prime}$ is finite.

With these properties of $\Lambda(\nu)$ we can now construct the solutions of Eq. (1.1). We will separate them into two classes.

\section{Class I-Discrete Eigenvalues}

Corresponding to the $2 n^{\prime}$ eigenvalues outside of the interval $[-\beta, \beta]$, one finds the $2 n^{\prime}$ solutions:

$$
\psi_{v_{i}}^{(0)}(x, \mu)=e^{-x / v_{i}} \frac{\nu_{i} f_{1}(\mu)}{\nu_{i}-\mu}, \quad \pm i=1,2, \cdots n^{\prime}
$$

where $\Lambda\left(\nu_{i}\right)=0$ for $\left(\nu_{i}\right)$ finite. As the $i$ th zero is $m_{i}$ degenerate, the $2 n^{\prime}$ eigenfunctions given by $(2.9)$ are supplemented by:

$$
\psi_{v_{i}}^{(j-1)}(x, \mu)=\left.\frac{d^{(j-1)}}{d v^{(j-1)}} \psi_{\nu}^{(0)}(x, \mu)\right|_{v=v_{i}} \quad \begin{aligned}
j & =2,3, \cdots m_{i} \\
\pm i & =1,2, \cdots n^{\prime}
\end{aligned}
$$

'The $2 N^{\prime}$ solutions given by (2.9) and (2.10) are linearly independent and by Eq. (2.4) clearly satisfy Eq. (1.1). For $x=0$, these eigenfunctions will be denoted by $\phi_{v_{i}}^{(j-1)}(\mu)$.

If $\int_{-\beta}^{\beta} f_{1}(\mu) f_{2}(\mu) d \mu=1$ then $\Lambda(\nu)$ has a double zero at infinity (assuming $\left.\int_{-\beta}^{\beta} \mu^{2} f_{1}(\mu) f_{2}(\mu) d \mu \neq 0\right)$. In this case Eq. (1.1) has the $2\left(N^{\prime}-1\right)$ solutions given by $(2.9)$ and $(2.10)$, and the two linearly independent solutions:

$$
\psi_{\infty}^{(1)}(x, \mu)=f_{1}(\mu) \text {, and } \psi_{\infty}^{(2)}(x, \mu)=f_{1}(\mu)[x-\mu] .
$$

\section{Class II-Continuum Solutions}

In addition to the $2 N^{\prime}$ solutions of Class I which correspond to the zeros of $\Lambda(\nu)$ there are the continuum of solutions:

$$
\psi_{\nu}(x, \mu)=e^{-x / \nu} \phi_{\nu}(\mu), \quad \text { for all } \nu-\beta \leqq \nu \leqq \beta .
$$

where

$$
\phi_{\nu}(\mu)=\nu f_{1}(\mu) P \frac{1}{\nu-\mu}+\lambda(\nu) \delta(\nu-\mu)
$$

corresponding to the branch cut of $\Lambda(\nu)$. From Eq. (2.3) one finds:

$$
\lambda(\nu) f_{2}(\nu)=\frac{\Lambda_{+}(\nu)+\Lambda_{-}(\nu)}{2} .
$$

It should be noted that Eq. (2.14) defines $\lambda(\nu)$ only up to delta functions in $v-\mu_{i}$, where $f_{2}\left(\mu_{i}\right)=0$. Therefore, implicit in the solutions of (2.12) are the solutions: 


$$
\psi_{\mu_{i}}(x, \mu)=e^{-x / \mu_{i}} \delta\left(\mu-\mu_{i}\right), \quad \text { all } i \text { such that } f_{2}\left(\mu_{i}\right)=0 .
$$

These will always be explicitly factored out of the solutions (2.12).

\section{COMPLETENESS THEOREM}

'I'HEOREM: The set of solutions of Class II for $\gamma_{1} \leqq \nu \leqq \gamma_{2}, \gamma_{1}<\gamma_{2},-\beta \leqq \gamma_{1}$, $\gamma_{2} \leqq \beta$, plus the solutions in Class I form a complete set of functions on the class of linear continuous functionals, $\psi(\mu)$, with compact support in $\left[\gamma_{1}, \gamma_{2}\right]$ and such that:

$$
\operatorname{Lim}_{\mu \rightarrow \gamma_{1}} \psi(\mu) \leqq C\left|\mu-\gamma_{2}\right|^{-\epsilon}, \quad \quad \epsilon<1
$$

and for either (or both) $\gamma_{1}, \gamma_{2}$ infinite

$$
\operatorname{Lim}_{\substack{\mu \rightarrow \gamma_{1} \\ \text { and } \mu_{1} \\ \gamma_{2} \rightarrow \infty}} \mu^{k} \psi(\mu) f_{2}(\mu)=0,
$$

for some $k$ to be specified later.

Both of the restrictions implied by (3.1) and (3.2) can be relaxed within the framework of distribution theory $(8)$; however, for convenience and simplicity this will not be done here.

To prove the theorem, we have to show that the singular integral equation

$$
\psi(\mu)=\sum_{i, j} a_{i j} \phi_{v_{i}}^{(j-1)}(\mu)+\int_{\gamma_{\perp}}^{\gamma_{2}} A(\nu) \phi_{v}(\mu) d \nu,
$$

has a solution when $\psi(\mu)$ is subject to the restrictions in the theorem. For the present it is required that both $\gamma_{1}$ and $\gamma_{2}$ be finite.

Letting $\psi^{\prime}(\mu)=\psi(\mu)-\sum_{i, j} a_{i j} \phi_{\nu_{i}}^{(j-1)}(\mu)$, Eq. (3.3) becomes:

$$
\psi^{\prime}(\mu)=f_{1}(\mu) P \int_{\gamma_{1}}^{\gamma_{2}} \frac{\nu A(\nu) d \nu}{\nu-\mu}+\lambda(\mu) A(\mu)+\sum_{i} b_{i} \delta\left(\mu-\mu_{i}\right) .
$$

Clearly the $b_{i}$ are given by:

$$
b_{i}=\operatorname{Lim}_{\epsilon \rightarrow 0} \int_{\mu_{i}-\epsilon}^{\mu_{i}+\epsilon} d \mu \psi(\mu), \quad \text { all } \gamma_{1} \leqq \mu_{i} \leqq \gamma_{2} .
$$

Assuming that $A(\nu)$ has the same properties as $\psi(\mu)$ the function

$$
N(z) \equiv \frac{1}{2 \pi i} \int_{\gamma_{1}}^{\gamma_{2}} \frac{\nu A(\nu) d \nu}{\nu}
$$

has the properties $(9)$ :

(a) $N(z)$ is analytic except on the interval $\left[\gamma_{1}, \gamma_{2}\right]$, and $N(z)$ has, at most, polar behavior in this interval. 
(b) $\operatorname{Lim}_{z \rightarrow \infty} N(z)=0$

(c) $\operatorname{Lim}_{z \rightarrow \gamma_{1}} N(z) \leqq C\left[z-\gamma_{2}\right]^{-\epsilon}$.

Multiplying Eq. (3.4) by $\mu f_{2}(\mu)$ and using (2.4) and (3.6) the expansion equation becomes the boundary value equation:

$$
\begin{gathered}
\gamma_{1} \leqq \mu \leqq \gamma_{2} \\
\mu f_{2}(\mu) \psi^{\prime}(\mu)=N_{+}(\mu) \Lambda_{+}(\mu)-N_{-}(\mu) \Lambda_{-}(\mu) .
\end{gathered}
$$

This equation can be put in the conventional form:

$$
\gamma(\mu) \psi^{\prime}(\mu)=N_{+}(\mu) X_{+}(\mu)-N_{-}(\mu) X_{-}(\mu),
$$

where

$$
\gamma(\mu)=\frac{\mu f_{2}(\mu) X_{-}(\mu)}{\Lambda_{-}(\mu)},
$$

and $X(z)$ has the following properties:

(a) $X(z)$ is sectionally holomorphic with boundary $\left(\gamma_{1}, \gamma_{2}\right)$.

(b) $X(z)$ is nonzero in the cut finite plane.

(c) $X_{+}(\mu) / X_{-}(\mu)=\Lambda_{+}(\mu) / \Lambda_{-}(\mu) \equiv G(\mu)$ on $\left(\gamma_{1}, \gamma_{2}\right)$.

(d) $X(z)$ vanishes more slowly than $\left|z-\gamma_{1}\right|$ and $\left|z-\gamma_{2}\right|$ as $z \rightarrow \gamma_{1}, \gamma_{2}$ respectively.

(e) $\operatorname{Lim}_{z \rightarrow \infty} X(z)=$ Class $(1)$ const.

Class (2) $z^{k_{1}}, k_{1}$ positive integer.

Class (3) $z^{-k_{2}}, k_{2}$ positive integer.

The problem of finding $X(z)$ subject to these conditions is the "classical" homogeneous Hilbert problem $(\tau)$. The solution is:

$$
\begin{aligned}
& X(z)=\left(z-\gamma_{1}\right)^{t_{1}}\left(z-\gamma_{2}\right)^{t_{2}} e^{\Gamma(z)} ; \\
& \Gamma(z)=\frac{1}{2 \pi i} \int_{\gamma_{1}}^{\gamma_{2}} \frac{\ln G(\mu) d \mu}{\mu-z} ;
\end{aligned}
$$

and $t_{1}$ and $t_{2}$ are integers chosen such that

$$
\begin{gathered}
1>t_{2}+\frac{6\left(\gamma_{2}\right)}{\pi} \geqq 0, \\
1>t_{1}-\frac{\theta\left(\gamma_{1}\right)}{\pi} \geqq 0 ; \quad \theta(\mu)=\arg \Lambda_{+}(\mu) .
\end{gathered}
$$

For the entire-space and half-space problems one can easily obtain $t_{1}$ and $t_{2}$ from Eq. (2.8). They are listed in Table I. 
TABLE I

Classification of the Functions $X(z)$

\begin{tabular}{ccccc}
\hline$\gamma_{1}$ & $\gamma_{2}$ & $t_{1}$ & $t_{2}$ & $t_{1}+t_{2}=-k_{2}$ \\
\hline$-\beta$ & $\beta$ & $-N^{\prime}$ & $-N^{\prime}$ & $-2 N^{\prime}$ \\
0 & $\beta$ & 0 & $-N^{\prime}$ & $-N^{\prime}$ \\
$-\beta$ & 0 & $-N^{\prime}$ & 0 & $-N^{\prime}$ \\
\hline
\end{tabular}

If $N^{\prime}>0$, then for the half-space and entire-space problem $X(z)$ belongs to the Class (3). Solving Eq. (3.8) for this class, we find:

$$
N(z)=\frac{1}{2 \pi i X(z)} \int_{\gamma_{1}}^{\gamma_{2}} \frac{d \mu \gamma(\mu) \psi^{\prime}(\mu)}{\mu-z},
$$

which is a solution to (3.4) only if the additional $k_{2}$ conditions are satisfied:

$$
\int_{\gamma_{1}}^{\gamma_{2}} \mu^{l} \gamma(\mu) \psi^{\prime}(\mu) d \mu=0, \quad l=0,1, \cdots k_{2}-1,
$$

To satisfy (3.14), $k_{2}$ discrete eigenfunctions are included in the sum contained in $\psi^{\prime}(\mu)$. As $k_{2} \leqq 2 N^{\prime}$, there are always enough eigenfunctions to do this. Even so, it still must be shown that Eq. (3.14) does not impose any restrictions on $\psi(\mu)$ other than those stated in the theorem. Using the representation of $X(z)$ derived in Appendix A, it will be shown that (3.14) is consistent with the theorem. Before proceeding it should be remarked that for $X(z)$ belonging to either Class (1) or (2), an $N(z)$ can be found which satisfies (3.4) without restrictions such as (3.14). In these cases no discrete eigenfunctions are needed and $A(\nu)$ is easily found to satisfy the assumed conditions. Therefore, in the following, only $X$ 's belonging to Class (3) will be considered.

Equation (3.14) is explicitly:

$$
\int_{\gamma_{1}}^{\gamma_{2}} \mu^{l} \gamma(\mu) \psi(\mu) d \mu=\sum_{i, j} a_{i j} \int_{\gamma_{1}}^{\gamma_{2}} \mu^{l} \gamma(\mu) \phi_{\nu_{i}}^{(j-1)}(\mu) d \mu
$$

$l=0,1, \cdots k_{2}-1$, and the sum is over $k_{2} a_{i j}$ 's. In order to express this equation in its simplest form, consider the integral:

$$
I_{l, i}^{(j-1)}=\int_{\gamma_{1}}^{\gamma_{2}} \mu^{l} \gamma(\mu) \phi_{\nu i}^{(j-1)}(\mu) d \mu
$$

which is

$$
I_{l, i}^{(j-1)}=\frac{d^{(j-1)}}{d \nu^{(j-1)}}-\left.\nu \int_{\gamma_{1}}^{\gamma_{2}} \frac{\mu^{l} \gamma(\mu) f_{1}(\mu) d \mu}{\mu-\nu}\right|_{\mu=\nu_{i}}
$$


Using the representation of $X(z)$ given by (A.3), (3.17) takes the form:

$$
I_{l, i}^{(j-1)}=\frac{d^{(j-1)}}{d \nu^{(j-1)}}-\left.\frac{\nu}{2 \pi i} \int_{0} \frac{z^{\prime} d z X(z)}{z-\nu}\right|_{v=v_{i}}
$$

( $C$ encircles the $\left(\gamma_{1}, \gamma_{2}\right)$ cut in the negative direction.) As the integrand goes at, most like $z^{-2}$ as $z$ goes to infinity, we have finally:

$$
I_{l, i}^{(j-1)}=\frac{d^{(j-1)}}{d \nu_{i}^{(j-1)}}-\nu_{i}^{l+1} X\left(\boldsymbol{\nu}_{i}\right) .
$$

If $\nu=\infty$ is a zero of $\Lambda(\nu)$, one may want or have to include $\phi_{\infty}^{(1)}(\mu)$ and $\phi_{\infty}^{(2)}(\mu)$ in the sum over the discrete solutions. In this casc the following integrals have to be simplified:

$$
I_{l, \infty}^{(0)} \equiv \int_{\gamma_{1}}^{\gamma_{2}} \mu^{l} \gamma(\mu) \phi_{\infty}^{(1)}(\mu) d \mu=\int_{\gamma_{1}}^{\gamma_{2}} \mu^{l} \gamma(\mu) f_{1}(\mu) d \mu
$$

and

$$
I_{l, \infty}^{(1)} \equiv \int_{\gamma_{1}}^{\gamma_{2}} \mu^{l} \gamma(\mu) \phi_{\infty}^{(2)}(\mu) d \mu=-\int_{\gamma_{1}}^{\gamma_{2}} \mu^{l+1} \gamma(\mu) f_{1}(\mu) d \mu .
$$

These integrals can be simply performed by using the representation of $X(z)$ given by (A.3) and letting $z \rightarrow \infty$. We find:

$$
\begin{aligned}
\int_{\gamma_{1}}^{\gamma_{2}} \mu^{l} \gamma(\mu) f_{1}(\mu) d \mu & =0 \quad l<k_{2}-1 \\
\int_{\gamma_{1}}^{\gamma_{2}} \mu^{k_{2}-1} \gamma(\mu) f_{1}(\mu) d \mu & =(-),
\end{aligned}
$$

and

$$
\int_{\gamma_{1}}^{\gamma_{2}} \mu^{k_{2}} \gamma(\mu) f_{1}(\mu) d \mu=B \equiv-\operatorname{Lim}_{z \rightarrow \infty} z\left[z^{k_{2}} X(z)-1\right] .
$$

Using the representation of $X(z)$ given by (3.10) and (3.11), B is found to be:

$$
B=\gamma_{1} t_{1}+\gamma_{2} t_{2}+\frac{1}{2 \pi i} \int_{\gamma_{1}}^{\gamma_{2}} \ln G(\mu) d \mu .
$$

It is now clear that the integrals $I_{l, i}^{(j-1)}$ are never zero for all $i$ and $j$. Therefore condition (3.14) can indeed be satisfied without further restrictions on $\psi(\mu)$. In Section IV it will be shown that the $a_{i j}$ 's are also uniquely determined.

For the entire-space problem we can construct $X(z)$ by inspection. The solution is given, up to a constant, by:

$$
X(z)=\frac{\Lambda(z)}{\prod_{i=1}^{n^{\prime}}\left(\nu_{i}^{2}-z^{2}\right)^{m_{i}}} .
$$


It has been specified that $\gamma_{1}$ and $\gamma_{2}$ be finite in the above procedure. It is clear that if $\beta=\infty$ the proof is still true with suitable restrictions on $\psi(\mu)$. For $\gamma_{1}=-\infty, \gamma_{2}=+\infty$, the solution for $X(z)$ is given by Eq. (3.23). If only one end point is finite, say $\gamma_{1}$, the end point condition for $X(z)$ is applied only to the finite end, $\gamma_{1} . X(z)$ is then given by:

$$
X(z)=\left(z-\gamma_{1}\right)^{t_{1}} e^{\Gamma^{(z)}} .
$$

and

$$
\Gamma(z)=\frac{1}{2 \pi i} \int_{\gamma_{1}}^{\infty} \frac{\ln G(\mu) d \mu}{\mu-z},
$$

where $\ln G(\mu)$ is that branch which vanishes at infinity. It is clear that $N(z)$ will exist with the correct properties, if in (3.2) we choose $k \geqq 2+k_{1}\left(k_{1}\right.$ given by Class (2)), or $k>2$, if $X(z)$ belongs to Class (1) or (2).

It is now easy to show that $A(\nu)$, given by $\nu A(\nu)=N_{+}(\nu)-N_{-}(\nu)$, does satisfy the assumed conditions. Therefore, the completeness theorem has been proven.

\section{APPLICATIONS TO HALF-SPACE PROBLEMS}

In this section the theorem of Section III will be used to obtain solutions to Eq. (1.1) for $0 \leqq x \leqq \infty$. For simplicity it is assumed that $N^{\prime}=n^{\prime}>0$; that $f_{2}(\mu) \neq 0$ for $0<\mu \leqq \beta$, except possibly at $\mu=\infty$ if $\beta=\infty$; and that $\nu_{i} \neq \infty$.

We first consider the albedo problem, where we want to find a solution of (1.1) for $0 \leqq x \leqq \infty$ subject to the boundary conditions:

$$
\psi(0, \mu)=\delta\left(\mu-\mu_{0}\right) \quad 0 \leqq \mu \leqq \beta, \quad 0<\mu_{0}<\beta
$$

and

$$
\operatorname{Lim}_{x \rightarrow+\infty} \psi(x, \mu)=0 .
$$

Using the theorem of Section III, we can write:

$$
\psi\left(x, \mu ; \mu_{0}\right)=\sum_{i} a_{i} \psi_{\nu i}^{(0)}(x, \mu)+\int_{0}^{\beta} A(\nu) \cdot \psi_{\nu}(x, \mu) d \mu .
$$

The sum is over $N^{\prime}$ discrete eigenfunctions and (4.1b) implies that the sum is over $i=1,2, \cdots N^{\prime}$. Condition (4.1a) gives:

$$
\begin{gathered}
0 \leqq \mu, \mu_{0} \leqq \beta \\
\delta\left(\mu-\mu_{0}\right)=\sum_{i=1}^{N^{\prime}} a_{i} \phi_{\nu_{i}}^{(0)}(\mu)+\int_{0}^{\beta} A(\nu) \phi_{\nu}(\mu) d \nu .
\end{gathered}
$$


The $a_{i}^{\prime}$ s can be determined by the $N^{\prime}$ conditions of (3.14). The equations are explicitly:

$$
\mu_{0}^{l} \gamma\left(\mu_{0}\right)=\sum_{i=1}^{N^{\prime}} a_{i} I_{l, i}^{(0)}
$$

It is easy to show that

$$
\operatorname{det} I_{l, i}^{(0)}=(-)^{N^{\prime}} \prod_{i=1}^{N^{\prime}} \nu_{i} X\left(\nu_{i}\right) \prod_{l>j=1}^{N^{\prime}}\left(\nu_{l}-\nu_{j}\right)
$$

which is clearly nonzero. Using Cramer's rule, the $a_{i}$ 's are found to be:

$$
a_{i}=-\frac{\gamma\left(\mu_{0}\right)}{\nu_{i} X\left(\nu_{i}\right)} \prod_{j \neq i}^{N^{\prime}}\left(\frac{\mu_{0}-\nu_{j}}{\nu_{i}-\nu_{j}}\right) \quad i=1,2, \cdots N^{\prime} .
$$

It should be mentioned that if $\nu_{i}$ is $m_{i}$ degenerate, $\left(N^{\prime} \neq n^{\prime}\right)$. Equation (4.4) becomes:

$$
\mu_{0}^{l} \gamma\left(\mu_{0}\right)=\sum_{i=1}^{N^{\prime}} b_{i} u_{i l}
$$

here

$$
\begin{aligned}
b_{j} & =a_{i j}, \\
& =a_{2, j-m_{1}}, \quad j=1,2, \cdots m_{1} \\
& j=m_{1}+1 \cdots m_{2}+m_{1}, \text { etc. }
\end{aligned}
$$

and

$$
\operatorname{det} u_{i j}=(-)^{N^{\prime}} \prod_{i=1}^{n^{\prime}}\left(\nu_{i} X\left(\nu_{i}\right)\right)^{m_{i}} \prod_{l>j=1}^{n^{\prime}}\left(\nu_{i}-\nu_{j}\right)^{m_{l} m_{j}},
$$

which is never zero and therefore the $a_{i j}$ 's can be uniquely determined in this case also.

In applications we normally want to know the discrete eigenfunction coefficients, because they usually give the asymptotic form of $\psi(x, \mu)$, and the emerging "angular" distribution: $\psi(0, \mu), \mu<0$. The emerging "angular" distribution for the albedo problem is:

$$
\psi\left(0, \mu ; \mu_{0}\right)=\sum_{i=1}^{N^{\prime}} \phi_{\nu_{i}}^{(0)}(\mu) a_{i}+\int_{0}^{\beta} A(\nu) \phi_{\nu}(\mu) d \nu, \quad \mu<0 .
$$

For $\mu<0, \phi_{\nu}(\mu)=\nu f_{1}(\mu) /(\nu-\mu)$, and Eq. (4.9) becomes:

$$
\psi\left(0, \mu ; \mu_{0}\right)=\sum_{i=1}^{N^{\prime}} a_{2} \phi_{\nu_{i}}^{(0)}(\mu)+2 \pi i f_{1}(\mu) N(\mu) .
$$


Expressing $N(\mu)$, for $\mu<0$ by using the representation of $X(z)$ given by Eq. (A.3), we obtain:

$$
N(\mu)=\frac{1}{2 \pi i X(\mu)}\left\{\frac{\gamma\left(\mu_{0}\right)}{\mu_{0}-\mu}+\sum_{i=1}^{N^{\prime}} \frac{a_{i} \nu_{i}}{\nu_{i}-\mu}\left[X(\mu)-X\left(\nu_{i}\right)\right]\right\} .
$$

The emerging "angular" distribution now reduces to:

$$
\begin{gathered}
\mu<0 ; \\
\psi\left(0, \mu ; \mu_{0}\right)=\gamma\left(\mu_{0}\right) \frac{f_{1}(\mu)}{\bar{X}(\mu)}\left[\frac{1}{\mu_{0}-\mu}-\sum_{i=1}^{N^{\prime}} \frac{1}{\nu_{i}-\mu} \prod_{j \neq i}\left(\frac{\mu_{0}-\nu_{j}}{\nu_{i}-\nu_{j}}\right)\right] .
\end{gathered}
$$

The Milne problem can be readily solved by using the formulas derived for the albedo problem. In the Milne problem we want to find the solution to Eq. (1.1) subject to the boundary conditions: $\left(\nu_{N}, \neq \infty\right)$

$$
\operatorname{Lim}_{x \rightarrow+\infty} \psi(x, \mu)=\psi_{-\nu_{i}}^{(0)}(x, \mu) \quad \operatorname{Re} \nu_{i}>0,
$$

and

$$
\psi(0, \mu)=0 \quad \mu \geqq 0 .
$$

Using the completeness theorem we can expand $\psi(x, \mu)$ subject to (4.13a) as:

$$
\psi(x, \mu)=\psi_{-\nu_{i}}^{(0)}(x, \mu)+\sum_{i=1}^{N^{\prime}} a_{i} \psi_{\nu_{i}}^{(0)}(x, \mu)+\int_{0}^{\beta} A(\nu) \psi_{\nu}(x, \mu) d \nu,
$$

and then $(4.13 \mathrm{~b})$ becomes:

$$
\begin{gathered}
\mu \geqq 0, \\
-\phi_{-\nu_{i}}^{(0)}(\mu)=\sum_{j=1}^{N^{\prime}} a_{j} \phi_{\nu_{j}}^{(0)}(\mu)+\int_{0}^{\beta} A(\nu) \phi_{\nu}(\mu) d \nu .
\end{gathered}
$$

In order to compute the $a_{i}$ 's and the emerging "angular" distribution we need only integrate the albedo solutions times $-\phi_{-v_{i}}\left(\mu_{\theta}\right)$ over $\mu_{0}$. This can again be done using (A.3), and we find:

$$
a_{k}=\frac{\nu_{i} X\left(-\nu_{i}\right)}{\nu_{k} X\left(\nu_{k}\right)} \prod_{j \neq k}\left(\frac{\nu_{i}+\nu_{j}}{\nu_{j}-\nu_{k}}\right), \quad k=1,2, \cdots N^{\prime}
$$

and the emerging "angular" distribution is:

$$
\mu<0
$$

$$
\psi(0, \mu)=\frac{X\left(-\nu_{i}\right)}{X(\mu)}\left[\phi_{-\nu_{i}}(\mu)+\nu_{i} f_{1}(\mu) \sum_{j=1}^{N^{\prime}} \frac{1}{\nu_{j}-\mu} \prod_{k \neq j}\left(\frac{\nu_{i}+\nu_{k}}{\nu_{k}-\nu_{j}}\right)\right] .
$$




\section{IDENTITIES FOR $X(z)$}

In Section IV we showed that the representation (A.3) for $X(z)$ allowed the usual cumbersome integrals involved in a half-space problem to be reduced to a form which involved only $X(z)$ and other, in principle, known functions. In this section we will derive two more identities for $X(z)$, both of which may serve as a means to numerically determine $X(z)$, or as will be shown by way of an example in Section VI, permit some quantities of interest to be trivially determined.

Defining $C=\int_{-\beta}^{\beta} f_{1}(\mu) f_{2}(\mu) d \mu$, and for convenience assuming $C \neq 1$, we want to prove:

$$
X(z) X(-z)=\frac{\Lambda(z)}{[1-C] \prod_{i=1}^{N^{\prime}}\left(\nu_{i}{ }^{2}-z^{2}\right)}, \quad\left(N^{\prime}=n^{\prime}\right)
$$

To prove this, consider the function

$$
R(z)=\frac{\Lambda(z)}{[1-C] X(z) X(-z) \prod_{i=1}^{N^{\prime}}\left(\nu_{i}^{2}-z^{2}\right)} .
$$

It is clear that $R(z)$ is analytic in the plane cut from $-\beta$ to $\beta$. Across the cut

$$
\frac{R_{+}(\mu)}{R_{-}(\mu)}=1 \text {, }
$$

and also

$$
\operatorname{Lim}_{z \rightarrow \infty} R(z)=1
$$

Therefore, $R(z)=1$, and the identity (5.1) is proved. From Eq. (5.1) we see immediately:

$$
X^{2}(0)=\frac{1}{[1-C] \prod_{i=1}^{N^{\prime}} \nu_{i}^{2}}
$$

The phase of $X(0)$ is uniquely determined by the fact that $\operatorname{Lim}_{z \rightarrow-\infty} X(z)=$ $(-)^{N^{\prime}}|z|^{-N^{\prime}}$ ( $z$ real), and that $X(z)$ is a nonzero, real function of $z$. Therefore

$$
X(0)=(-)^{N^{\prime}}\left[\frac{1}{(1-C) \prod_{i=1}^{N^{\prime}}{\nu_{i}^{2}}^{2}}\right]^{1 / 2} .
$$

A particularly simple result of this is that $[1-C] \prod_{i=1}^{N^{\prime}} \nu_{i}^{2}$ must be real and nonnegative. Of course, if $C=1$, an identity similar to $(5.1)$ can be derived.

The identity (5.1) may be useful for calculating $X(z)$, for large $z$. Expanding 
both sides of Eq. (5.1) in powers of $z^{-1}$, and equating coefficients, one finds a set of coupled algebraic equations for the coefficients of $z^{-n}$ in $X(z)$. In particular cases a truncated solution may rapidly converge and the coefficients of the low inverse powers of $z$ be easily obtainable.

To obtain the second useful identity for $X(z)$ we use the representation of $X(z)$ given by (A.3) and the identity (5.1). We find:

$$
X(z)=\frac{1}{[1-C]} \int_{-\beta}^{0} \frac{d \mu}{(\mu+z)} \frac{\mu f_{1}(\mu) f_{2}(\mu)}{X(\mu) \prod_{i=1}^{N^{\prime}}\left(\nu_{i}{ }^{2}-\mu^{2}\right)} .
$$

From (5.5) we first notice that if we know $X(\mu)$ for $-\beta \leqq \mu \leqq 0$, we can readily determine $X(z)$ everywhere. Such being the case, by letting $z=\mu$, $-\beta \leqq \mu \leqq 0$ in (5.5) we obtain a nonlinear integral equation for $X(\mu)$, $-\beta \leqq \mu \leqq 0$, for which an iterative solution may rapidly converge. More important is the property that (5.5) allows us to express integrals over $X(\mu)^{-1}$ (which always appear in the emerging "angular" distribution), in terms of $X(z)$, and it is this property that we will exploit in the next section.

\section{AN APPLICATION}

To illustrate the usefulness of the identities derived in the preceding section we will consider the model of a one-dimensional plasma with a uniform background of ions confined to the half-space, $x>0$, by a diffuse reflecting wall. In the usual linear Vlasov approximation the governing equations are:

$$
\frac{\partial f(x, v, t)}{\partial t}+v \frac{\partial}{\partial x}=\frac{e n E}{m} \frac{\partial f_{0}(v)}{\partial v}
$$

and

$$
\frac{\partial E^{\prime}(x, t)}{\partial x}=-4 \pi e \int_{-\infty}^{\infty} f(v, x, t) d v
$$

Here

$$
f(x, v, t)=\int_{-\infty}^{\infty} f(x, \mathbf{v}, t) d v_{z} d v_{y}
$$

$n$ is total number of electrons, $f$ is the deviation of the electron distribution function from the equilibrium distribution $n f_{0}$, and $f_{0}$ is normalized to one. For convenience $f_{0}$ will be chosen Maxwellian, i.e.,

$$
f_{0}(v)=\left(\frac{\beta m}{2 \pi}\right)^{1 / 2} e^{-\beta m v^{2} / 2} .
$$


Introducing Fourier transforms in time,

$$
\tilde{f}(v, x)=(2 \pi)^{-1 / 2} \int_{-\infty}^{\infty} f(x, v, t) e^{i \omega t} d t
$$

and looking for eigenfunctions of the form:

$$
\tilde{f}(v, x)=e^{i \omega x / \nu} \phi_{\nu}(v) \text {, and } \tilde{E}(x)=e^{i \omega x / \nu} E_{\nu},
$$

Eqs. (6.1) become:

$$
\begin{aligned}
(\nu-v) \phi_{\nu}(v) & =\nu^{2} g_{1}(v) \int_{-\infty}^{\infty} \phi_{\nu}\left(v^{\prime}\right) d v^{\prime}, \\
E_{\nu} & =-\frac{4 \pi e \nu}{i \omega} \int_{-\infty}^{\infty} \phi_{\nu}\left(v^{\prime}\right) d v^{\prime},
\end{aligned}
$$

where

$$
g_{1}(v)=-\frac{\omega_{p}^{2}}{\omega^{2}} \frac{\partial f_{0}(v)}{\partial v}, \quad \omega_{p}^{2}=\frac{4 \pi e^{2} n}{m} .
$$

In addition to solutions of the form (6.4) there is another solution of (6.1), namely:

$$
\widetilde{E}=\text { constant }=E_{0}
$$

and the corresponding electron distribution function:

$$
\tilde{f}^{(0)}(v)=-\frac{e E_{0} n}{i \omega n} \frac{\partial f_{0}(v)}{\partial v} .
$$

Even though Eq. (6.5a) is not of the exact form as (2.2), with trivial modification the completeness theorem is still true when the additional solution (6.6) is included. Just as in Section II, the discrete eigenvalues of the Eqs. (6.5) are given by the zeros of $\Lambda(\nu)$, where

$$
\Lambda(\nu)=1+\nu^{2} \int_{-\infty}^{\infty} \frac{g_{1}(v) d v}{v-\nu} .
$$

Noting that

$$
\operatorname{Lim}_{\nu \rightarrow \infty} \Lambda(\nu)=1-\frac{\omega_{p}^{2}}{\omega^{2}}\left(1+\frac{\overline{3 v^{2}}}{\nu^{2}}+\cdots\right),
$$

and that the $\operatorname{Im} \Lambda_{+}(\nu)=0$ only for $\nu=0, \pm \infty, \mathrm{Eq}$. (6.8) gives:

and

$$
N^{\prime}=1 \text { if } \omega^{2} \leqq \omega_{p}^{2},
$$

$$
N^{\prime}=0 \text { if } \omega^{2}>\omega_{p}^{2} \text {. }
$$


As the time dependent problem will not be considered here, we will assume the simplest case, when $\omega^{2}>\omega_{p}{ }^{2}$.

The continuum solutions of (6.5) corresponding to the branch cut of $\Lambda(\nu)$ are:

$$
\phi_{\nu}(v)=\nu^{2} g_{1}(v) P \frac{1}{\nu-v}+\lambda(\nu) \delta(\nu-v)
$$

and

$$
\lambda(\nu)=\frac{\Lambda_{+}(\nu)+\Lambda_{-}(\nu)}{2} .
$$

In the diffuse reflecting wall problem we have a plasma confined to the region $x>0$, by a nonelectromagnetic wall which absorbs electrons from the plasma and re-emits electrons incoherently back into the plasma. The boundary conditions can be chosen so that $\widetilde{E}(0)=\widetilde{E}(x=0)$ is given and for

$$
v>0, \quad \tilde{f}(0, v)=-h(v) \int_{-\infty}^{0} v \tilde{f}(0, v) d v \equiv \operatorname{Ch}(v) .
$$

Requiring

$$
\int_{0}^{\infty} v h(v) d v=1
$$

automatically implies that the electron current is conserved at the wall;

$$
\int_{-\infty}^{\infty} \tilde{f}(0, v) v d v=0
$$

which must be satisfied for this problem.

Using the radiation condition, $\tilde{f}(x, v)$ and $\widetilde{E}(x)$ have the following expansion:

$$
\begin{gathered}
\tilde{f}(x, v)=\int_{0}^{\infty} A(\nu) e^{i \omega x / \nu} \phi_{\nu}(v) d \nu-\frac{e n E_{0}}{m i \omega} \frac{\partial f_{0}(v)}{\partial v} \\
\tilde{E}(x)=\int_{0}^{\infty} A(\nu)\left(-\frac{4 \pi e \nu}{i \omega}\right) e^{i \omega x / \nu} d \nu+E_{0} .
\end{gathered}
$$

At $x=0$, the boundary conditions give us:

$$
v>0 \quad C h(v)=\int_{0}^{\infty} A(\nu) \phi_{\nu}(v) d \nu-\frac{e n E_{0}}{m i \omega} \frac{\partial f_{0}}{\partial v}
$$

and

$$
\widetilde{E}(0)=\int_{0}^{\infty} A(\nu)\left(-\frac{4 \pi e \nu}{i \omega}\right) d \nu+E_{0} .
$$


Assuming $C$ is known, Eqs. (6.15) can be solved for $E_{0}$, and $A(\nu)$ just as in the preceding sections. If we let

$$
N(z)=\frac{1}{2 \pi i} \int_{0}^{\infty} \frac{\nu^{2} A(\nu) d \nu}{\nu-z},
$$

we find:

$$
N(z)=\frac{1}{2 \pi i X(z)} \int_{0}^{\infty} \frac{\gamma(v) h^{\prime}(v) d v}{v-z}
$$

where

$$
\begin{aligned}
& h^{\prime}(v)=v^{2} C h(v)+\frac{e v^{2} n E_{0}}{m i \omega} \frac{\partial f_{0}}{\partial v}, \\
& X(z)=\exp \left(\frac{1}{\pi} \int_{0}^{\infty} \frac{\theta(v) d v}{v-z}\right), \quad \theta(v)=\arg \Lambda_{+}(v),
\end{aligned}
$$

and

$$
\gamma(v)=\frac{X_{-}(v)}{\Lambda_{-}(v)} .
$$

To find $C$ and $E_{0}$ explicitly we have to find the electron current emerging from the plasma at $x=0$ by using the above solutions to (6.15). To do this we need the identities for the $X(z)$ given by (6.19). Noting that now, $\operatorname{Lim}_{x \rightarrow \infty} X(z)=1$, we obtain as before:

$$
\begin{aligned}
X(z) & =1+\int_{0}^{\infty} \frac{v^{2} g_{1}(v) \gamma(v) d v}{v-z} \\
X(z) X(-z) & =\frac{\Lambda(z)}{1-\left(\omega_{p}^{2} / \omega^{2}\right)}
\end{aligned}
$$

and

$$
X(z)=1+\frac{1}{1-\left(\omega_{p}^{2} / \omega^{2}\right)} \int_{-\infty}^{0} \frac{d v v^{2} g_{1}(v)}{X(v)(v+z)} .
$$

The velocity distribution function for electrons emerging from the plasma is given by:

$$
\begin{gathered}
v<0 \\
\tilde{f}(0, v)=2 \pi i N(v) g_{1}(v)-\frac{e n E_{0}}{m i \omega} \frac{\partial f_{0}(v)}{\partial v}
\end{gathered}
$$


Using Eq. (6.21), $N(z)$ given by Eq. (6.17) simplifies and the "emerging" distribution becomes:

$$
\begin{gathered}
v<0 \\
f(0, v)=\frac{g_{1}(v)}{\bar{X}(v)} C \int_{0}^{\infty} \frac{\gamma\left(v^{\prime}\right) v^{\prime 2} h\left(v^{\prime}\right) d v^{\prime}}{v^{\prime}-v}-\frac{i e n E_{0} \omega g_{1}(v)}{m \omega_{p}{ }^{2} X(v)} .
\end{gathered}
$$

Now, using $C=-\int_{-\infty}^{0} v \tilde{f}(0, v) d v$, and $(6.25)$ we obtain:

$-C=-C \int_{0}^{\infty} \gamma\left(v^{\prime}\right) v^{\prime 2} h\left(v^{\prime}\right) d v^{\prime} \int_{-\infty}^{0} \frac{g_{1}(v) v d v}{X(v)\left(v-v^{\prime}\right)}$

$$
-\frac{i e n E_{0} \omega}{m \omega_{p}^{2}} \int_{-\infty}^{0} \frac{g_{1}(v) v d v}{X(v)} .
$$

The integral in the second term of $(6.26)$ is clearly just $(X(0)-1)$. $\left[1-\left(\omega_{p}^{2} / \omega^{2}\right)\right]$. The second integral in the first term of $(6.26)$ is just

$$
\left(1-\frac{\omega_{p}^{2}}{\omega^{2}}\right) \frac{\left[X\left(-v^{\prime}\right)-X(0)\right]}{v^{\prime}} \text {. }
$$

Using Eqs. (6.22) and (6.12), (6.26) finally becomes:

$$
C X(0) \int_{0}^{\infty} d v \gamma(v) v h(v)=\frac{i e n E_{0}^{\prime} \omega}{m \omega_{p}^{2}}[X(0)-1] .
$$

Noting the fact that

$$
\tilde{E}(0)=-\frac{4 \pi e}{i \omega}(2 \pi i N(0))+E_{0},
$$

expressing $N(0)$ in its simplest form, and then solving (6.27) and (6.28) for $C$ and $E_{0}$ we obtain ${ }^{1}$ :

$$
E_{0}=E(0)\left(1-\frac{\omega_{p}^{2}}{\omega^{2}}\right)^{-1}
$$

and

$$
C=\frac{i \omega E(0) X(0)[X(0)-1]}{4 \pi e \int_{0}^{\infty} d v v h(v) \gamma(v)} .
$$

From $(6.22)$ we see that

$$
X(0)=\left(1-\frac{\omega_{p}^{2}}{\omega^{2}}\right)^{-1 / 2}
$$

${ }^{1}$ This result is more simply obtained by using the continuity equation in Poisson's equation and using $\operatorname{Lim}_{x \rightarrow \infty} \widetilde{E}(x)=E_{0}$. 
so the only quantity left to be determined is the integral in the denomination of $(6.30)$. If we consider two extremes; the wall re-emitting electrons at the temperature of the unperturbed plasma; and the wall at zero temperature, this integral can be easily done.

For the wall at the temperature of the unperturbed plasma, the emitting distribution function which conserves electron current is simply:

$$
h(v)=\beta m e^{-\beta m v^{2} / 2} .
$$

For this distribution we find:

$$
\int_{0}^{\infty} v d v h(v) \gamma(v)=\frac{\omega^{2}}{\omega_{p}^{2}} X^{\prime}(0)\left(\frac{2 \pi}{m \beta}\right)^{1 / 2},
$$

and the current of electrons entering the wall; $-C$, is then:

$$
-C=\frac{i e n E(0) X(0)}{\omega}\left(\frac{\beta}{2 \pi m}\right)^{1 / 2} \frac{[1-X(0)]}{\bar{X}^{\prime}(0)} .
$$

For the wall at zero temperature the emitting distribution function can be chosen:

$$
h(v)=-\operatorname{Lim}_{v_{v} \rightarrow 0} \delta^{\prime}\left(v-v_{0}\right) .
$$

The current of electrons entering the wall is now simply:

$$
-C=\frac{i \omega E(0)}{4 \pi e}[1-X(0)] \text {. }
$$

One can similarly calculate the density of perturbed electrons,

$$
\tilde{\rho}(0)=\int_{-\infty}^{\infty} f(0, v) d v
$$

at the wall. For the wall at the equilibrium temperature we obtain:

$$
\tilde{\rho}(0)=\frac{i \omega E(0)}{4 \pi e}\left[\frac{(X(0)-1)}{X^{\prime}(0)} \frac{X^{\prime \prime}(0)}{2}+\frac{X^{\prime}(0)}{\bar{X}(0)}\right]
$$

and for the wall at zero temperature,

$$
\tilde{\rho}(0)=\frac{i \omega E(0)}{4 \pi e} X^{\prime}(0) .
$$

We have thus seen that the identity relations for $X(z)$ have allowed us to express several quantities of interest in terms of rather simple functions. Of course, more detailed information about the system will in general be more complicated, but even then these identities will help simplify the formulas. 


\section{CONCLUSION}

While we have considered only equations of the form (1.1) and the slightly different equations of the plasma problem it is clear that for any equation or system of equations which have a spectrum similar to Eq. (1.1) and for which a completeness theorem of the form of Section III is valid there are similar identities for the solution of the associated homogeneous Hilbert problem for the half-space. The judicious use of these identities will always simplify the formulas of interest.

\section{APPENDIX A}

If $X(z)$ belongs to the Class (3), i.e., $\operatorname{Lim}_{z \rightarrow \infty} X(z)=z^{-k_{2}}, k_{2}>0$, and integer, we have by Cauchy's theorem.

$$
X(z)=\frac{1}{2 \pi i} \int_{C} \frac{X\left(z^{\prime}\right) d z^{\prime}}{z^{\prime}-z}
$$

where $C$ encircles the $\left(\gamma_{1}, \gamma_{2}\right)$ cut in the negative direction. Equation (A.1) then becomes:

$$
\begin{aligned}
X(z)=\frac{1}{2 \pi i} \int_{\gamma_{1}}^{\gamma_{2}} \frac{d \mu}{\mu-z}\left[X_{+}(\mu)\right. & \left.-X_{-}(\mu)\right] \\
= & \frac{1}{2 \pi i} \int_{\gamma_{1}}^{\gamma_{2}} \frac{d \mu X_{-}(\mu)}{(\mu-z) \Lambda_{-}(\mu)}\left[\Lambda_{+}(\mu)-\Lambda_{-}(\mu)\right],
\end{aligned}
$$

or

$$
X(z)=\int_{\gamma_{1}}^{\gamma_{2}} \frac{d \mu \gamma(\mu) f_{1}(\mu)}{\mu-z} .
$$

If $X(z)$ is not in the Class (3), a similar representation of $X(z)$ can be derived by making suitable substractions.

Received July 5, 1962

\section{REFERENCES}

1. K. M. CAse, Ann. Phys. (N.Y.) 9, 1 (1960).

2. K. M. CASE, Ann. Phys. (N.Y.) 7, 349 (1959).

3. C. Cercignani, Ann. Phys. (N.Y.) 20, 219 (1962).

4. F. ShURe, University of Michigan Thesis.

5. H. S. Wang, Natl. Bureau of Standards, Report (I).

6. R. S. Zelazny, Case Inst. of Tech., Report No. A.6.

7. N. I. Muskimelishvili, "Singular Integral Equations." Noordhoff, Groningen, 1953.

8. I. M. Gelfand and G. E. Schilow, "Verallgemeinerte Funktionen (Distributionen)," Vol. I. DVW, Berlin, 1960.

9. H. J. Bremmermann and L. Durand, J. Math. Phys. 2, 240 (61). 